\title{
Erratum to: Multi-party quantum key agreement with Bell states and Bell measurements
}

\author{
Run-Hua Shi · Hong Zhong
}

Received: 6 July 2012 / Accepted: 9 July 2012 / Published online: 25 July 2012

(C) Springer Science+Business Media, LLC 2012

\section{Erratum to: Quantum Inf Process DOI 10.1007/s11128-012-0443-2}

The title of "Multi-party quantum key agreement with bell states and bell measurements" should be "Multi-party quantum key agreement with Bell states and Bell measurements". We regret this error.

The online version of the original article can be found under doi:10.1007/s11128-012-0443-2.

R.-H. Shi $(\bowtie) \cdot$ H. Zhong

Key Laboratory of Intelligent Computing \& Signal Processing of Ministry of Education, Anhui University, Hefei 230039, China

e-mail: hfsrh@sina.com

R.-H. Shi · H. Zhong

School of Computer Science and Technology, Anhui University, Hefei 230039, China 